PROCEEDINGS OF THE

AMERICAN MATHEMATICAL SOCIETY

Volume 130, Number 9, Pages 2599-2607

S 0002-9939(02)06551-6

Article electronically published on April 17, 2002

\title{
A NOTE ON BESOV REGULARITY OF LAYER POTENTIALS AND SOLUTIONS OF ELLIPTIC PDE'S
}

\author{
MARIUS MITREA
}

(Communicated by Andreas Seeger)

\begin{abstract}
Let $L$ be a second order, (variable coefficient) elliptic differential operator and let $u \in B_{\alpha}^{p, p}(\Omega), 1<p<\infty, \alpha>0$, satisfy $L u=0$ in the Lipschitz domain $\Omega$. We show that $u$ can exhibit more regularity on Besov scales for which smoothness is measured in $L^{\tau}$ with $\tau<p$. Similar results are valid for functions representable in terms of layer potentials.
\end{abstract}

\section{Introduction AND STATEMENT OF RESUlts}

Let $\Omega$ be a bounded Lipschitz domain in $\mathbb{R}^{d}$ (cf. [12]) and, for each $h \in \mathbb{R}^{d}$, denote by $\Omega_{h}$ the collection of all $x \in \Omega$ with the property that the line segment $[x, x+h]$ is contained in $\Omega$. Then the modulus of smoothness of a function $u \in L^{p}(\Omega)$, $0<p<\infty$, is defined by

$$
\omega_{r}(u, t)_{L^{p}(\Omega)}:=\sup \left\{\left\|\Delta_{h}^{r}(u, \cdot)\right\|_{L^{p}\left(\Omega_{r h}\right)} ;|h| \leq t\right\}, \quad t>0,
$$

where $\Delta_{h}^{r}$ stands for the $r$-th difference with step $h$. Then, for $\alpha>0$ and $0<p, q<$ $\infty$, the Besov space $B_{\alpha}^{p, q}(\Omega)$ is introduced as the space of all functions $u$ for which

$$
\|u\|_{B_{\alpha}^{p, q}(\Omega)}:=\|u\|_{L^{p}(\Omega)}+\left(\int_{0}^{\infty}\left[t^{-\alpha} \omega_{r}(u, t)_{L^{p}(\Omega)}\right]^{q} d t / t\right)^{1 / q}<+\infty,
$$

where $r:=[\alpha]+1$. For more on this the reader is referred to [4]. Here we only want to make the important observation that this definition coincides with the one based on the Fourier transform and Littlewood-Paley theory as in, e.g., 1] p. 140 provided that $\alpha / d>\max \{0,1 / p-1\}$. See [13] p. 41 for a proof.

The main concern for us here is a certain 'smoothing' phenomenon, first observed in the context of harmonic Besov spaces in 2. More specifically, it has been shown in [2] that if $u \in B_{\lambda}^{p, p}(\Omega)$ with $1<p<\infty, \lambda>0$, is a harmonic function, then also

$$
u \in B_{\alpha}^{\tau, \tau}(\Omega) \text { for each } \lambda \leq \alpha<\lambda d /(d-1) \text {, if } 1 / \tau=1 / p+\alpha / d .
$$

Thus, harmonic functions in Besov spaces could exhibit more smoothness, when considered on appropriate, alternative scales (note that this is accomplished at the expense of lowering the integrability exponent). This phenomenon is significant when dealing with the issue of regularity for boundary-value problems for Laplace's

Received by the editors March 16, 2001.

2000 Mathematics Subject Classification. Primary 35B65, 31B10; Secondary 42B20, 46 E35.

Key words and phrases. Besov space regularity, Lipschitz domains, layer potentials, smoothness, elliptic PDE.

The author was partially supported by NSF grant DMS-9870018. 
equation, as well as for determining the efficiency of certain nonlinear approximation methods (such as those involving wavelet-based Galerkin schemes). See [2], [3], for a more extensive discussion in this regard.

The aim of this note is to investigate similar phenomena in the context of more general PDE's. Here we deal with null-solutions of (variable coefficient) elliptic, second order differential operators, and with functions representable by means of layer potentials. In order to state our main results, consider a smooth, compact, boundaryless, Riemannian manifold $M$, of real dimension $d$. Also, let $L$ be a second order, elliptic differential operator in $M$ locally given by

$$
L u=\sum_{j, k} \partial_{j} A^{j k} \partial_{k} u+\sum_{j} B^{j} \partial_{j} u-V u
$$

where $A^{j k}:=\left(a_{j k}^{\alpha \beta}\right)_{\alpha, \beta}, B^{j}:=\left(b_{j}^{\alpha \beta}\right)_{\alpha, \beta}$ and $V:=\left(v^{\alpha \beta}\right)_{\alpha, \beta}$ are matrix-valued functions with entries satisfying

$$
a_{j k}^{\alpha \beta} \in \operatorname{Lip}, \quad b_{j}^{\alpha \beta} \in L_{1}^{p^{*}}, \quad v^{\alpha \beta} \in L^{p^{*}}, \text { for some } p^{*}>d .
$$

Here and elsewhere, $L_{s}^{p}, 1<p<\infty, s \in \mathbb{R}$, will stand for the usual Sobolev scale. For definitions and basic properties the reader is referred to [6].

Throughout this note, for each point $\left(x_{0}, y_{0}\right)$ in the first quadrant in $\mathbb{R}^{2}$, we set $\Theta^{d}\left(x_{0}, y_{0}\right)$ for the collection of all points $(x, y)$ such that either $y_{0} \leq y<1+x / d$ and $y-y_{0}+x_{0}>x>0$, or $0<y<y_{0}$ and $d\left(y-y_{0}\right)+x_{0} \geq x>0$. In other words, $\Theta^{d}\left(x_{0}, y_{0}\right) \subset \mathbb{R}^{2}$ is the polygonal region in the first quadrant bordered by the lines passing through $\left(x_{0}, y_{0}\right)$ and having slopes 1 and $1 / d$, and the line passing through $(0,1)$ and having slope $1 / d$.

Theorem 1.1. Let $\Omega$ be a Lipschitz subdomain of $M$, and let $L$ be as in (1.4) (1.5). Next assume that $0<\alpha<1, p^{*} /\left(p^{*}-1\right)<p<p^{*}$, and that $u \in B_{\alpha}^{p, p}(\Omega)$ satisfies $L u=0$ in $\Omega$. Then also $u \in B_{\gamma}^{\tau, \tau}(\Omega)$, provided

$$
(\gamma, 1 / \tau) \in \Theta^{d}(\alpha, 1 / p) \text { and } 2-\alpha>\frac{1}{\tau}-\frac{1}{p}
$$

Moreover, if (1.5) is strengthened to

$$
a_{j k}^{\alpha \beta}, b_{j}^{\alpha \beta} \in C^{1+\varepsilon}, \quad \text { some } \varepsilon>0, \quad v^{\alpha \beta} \in L^{\infty},
$$

then the same conclusion holds for $1<p<\infty$.

What makes this type of result nontrivial is the fact that the source of the extra smoothness for $u$ is the fulfillment of the partial differential equation $L u=0$. For maximum applicability it is important to allow nonsmooth domains and operators whose coefficients have only a limited amount of smoothness. Note that the vectorvalued case is covered as well.

To state our next result, let $D u:=\sum a_{j} \partial_{j} u+b u$ be an elliptic, first-order differential operator on $M$, whose (matrix-valued) coefficients are assumed to satisfy

$$
a_{j}^{\alpha \beta} \in L_{2}^{p^{*}}, \quad b^{\alpha \beta} \in L_{1}^{p^{*}} \quad \text { for some } p^{*}>d=\operatorname{dim} M .
$$

Note that if all metrics involved are of class $L_{2}^{p^{*}}$, then the coefficients of $D^{*}$, the formal adjoint of $D$, also satisfy (1.8). In particular,

$$
L:=-D^{*} D
$$


is a formally self-adjoint, strongly elliptic operator whose coefficients, in the writing (1.4), satisfy

$$
a_{j k}^{\alpha \beta} \in L_{2}^{p^{*}}, \quad b_{j}^{\alpha \beta} \in L_{1}^{p^{*}}, \quad v^{\alpha \beta} \in L^{p^{*}} .
$$

The operator $D$ is said to have the unique continuation property (abbreviated $D \in$ UCP henceforth) if

$$
u \in L_{1}^{2}(M), D u=0 \text { on } M \Rightarrow \operatorname{supp} u \text { is either } M \text { or empty. }
$$

Theorem 1.2. Let $\Omega$ be a Lipschitz subdomain of $M$, and let $L$ satisfy either of the following two conditions:

(i) $L$ is as in (1.8)-(1.10), where $D \in \mathrm{UCP}$;

(ii) $L$ is a formally self-adjoint, second-order, strongly elliptic differential operator whose coefficients satisfy (1.4)-1.5.

Also, assume that $u \in L_{s+\frac{1}{p}}^{p}(\Omega)$ satisfies $L u=0$ in $\Omega$. Then, if $p^{*} /\left(p^{*}-1\right)<p<p^{*}$, $0<s<1$,

$$
u \in B_{\gamma}^{\tau, \tau}(\Omega) \text { provided }(\gamma, 1 / \tau) \in \Theta^{d}(s+1 / p, 1 / p),
$$

provided either $L$ is as in $(i)$ above, or $p=2$ and $L$ is as in (ii) above. The same conclusion holds for $1<p<\infty$ granted that $L$ is as in $(i)$ above and its coefficients satisfy (1.7).

Furthermore, when $p=2$ and $s \in\{0,1\}$, then also

$$
u \in B_{s+1 / q}^{q, 2}(\Omega) \text { for each } 1<q \leq 2 .
$$

A few comments are in order here.

Remarks. (i) An important case when Theorem 1.2 applies occurs for $D=\operatorname{grad}$ on a Riemannian manifold $M$, which entails $L=\Delta$, the Laplace-Beltrami operator on $M$.

(ii) At the level of Dirac operators (with reasonably smooth coefficients; cf. [9] for precise conditions) $D$ on $M$, we have

$$
\begin{aligned}
& u \in B_{s+1 / p}^{p, p}(\Omega), 0<s<1,1<p<\infty, D u=0 \text { in } \Omega \Longrightarrow \\
& u \in B_{\gamma}^{\tau, \tau}(\Omega) \text { for each }(\gamma, 1 / \tau) \in \Theta^{d}(s+1 / p, 1 / p) .
\end{aligned}
$$

(iii) Finally, Theorem 1.2 (as well as the just mentioned extensions) can be used in concert with Theorem 1.1 to produce further new regularity results. We leave the details to the reader.

Next, turning attention to integral operators, assume that the kernel $k(x, y)$ is defined on $M \times M \backslash$ diagonal. Then, for a (fixed) domain $\Omega$ in $M$ consider the (layer potential type) operator

$$
\mathcal{K} f(x):=\left\langle\left. k(x, \cdot)\right|_{\partial \Omega}, f\right\rangle, \quad x \in \Omega,
$$

where $\langle\cdot, \cdot\rangle$ stands for the natural pairing between classes of distributions and their corresponding test functions on $\partial \Omega$. Finally, $B_{s}^{p, q}(\partial \Omega), 1<p, q<\infty, s \in \mathbb{R}$, will denote the usual Besov scale on $\partial \Omega$; cf., e.g., [6]. 
Theorem 1.3. Let $\Omega$ be a Lipschitz domain in $M$ and assume that, for a positive integer $N$ and some non-negative integer $\theta$, the kernel $k(x, y)$ satisfies

$$
\left|\nabla_{x}^{i} \nabla_{y}^{j} k(x, y)\right| \leq C \operatorname{dist}(x, y)^{-(d-1-\theta+i+j)}, \quad \forall i=0,1, \ldots, N, \forall j=0,1,
$$

where the constant $C>0$ is independent of $x, y$. Also, recall the integral operator $\mathcal{K}$ associated with $k(x, y)$ as in (1.15). Then the operator

$$
\mathcal{K}: B_{-s}^{p, p}(\partial \Omega) \longrightarrow B_{\gamma}^{\tau, \tau}(\Omega)
$$

is bounded in each of the following situations:

$$
\begin{aligned}
& 0<s<1, \quad 1<p<\infty, \quad \theta \geq 1 \text { and } \\
& N>1-s+\frac{1}{\tau}, \quad(\gamma, 1 / \tau) \in \Theta^{d}(\theta+1 / p-s, 1 / p)
\end{aligned}
$$

or

$$
\begin{aligned}
& 0<s<1 / p<1, \quad \theta=0 \quad \text { and } \\
& N>1-s+\frac{1}{\tau}, \quad(\gamma, 1 / \tau) \in \Theta^{d}(1 / p-s, 1 / p) .
\end{aligned}
$$

A natural class of functions $k(x, y)$ for which (1.16) holds consists of Schwartz kernels of pseudodifferential operators of order $-1-\theta$ on $M$. In this latter case, we also have the following result.

Theorem 1.4. Let $k(x, y)$ denote the Schwartz kernel of a (classical) pseudodifferential operator $P \in O P S^{-1-\theta}(M)$ and let $\mathcal{K}$ be associated with $k(x, y)$ as in (1.15). Assume that the principal symbol $\sigma(P ; x, \xi)$ is even in $\xi$ when $\theta$ is odd, and odd in $\xi$ when $\theta$ is even. Then, for each $0 \leq s \leq 1$, the operator

$$
\mathcal{K}: L_{-s}^{2}(\partial \Omega) \longrightarrow B_{\gamma}^{\tau, \tau}(\Omega)
$$

is bounded provided

$$
(\gamma, 1 / \tau) \in \Theta^{d}(\theta-s+1 / 2,1 / 2), \quad \tau>1, \quad \theta \geq 1
$$

The same conclusion also holds when $\theta=0$ and $s=0$.

These regularity results are valid for large classes of PDE's in Lipschitz domains, including the Hodge Laplacian (on a manifold whose metric has only a limited amount of smoothness), as well as the Lamé and the Stokes system. Also, via standard methods, they can be easily extended to encompass (inhomogeneous) Poisson type problems for such systems.

\section{Proofs}

We debut with a result inspired by the work in [2]. Recall that $\operatorname{dim} M=d$.

Proposition 2.1. Let $\Omega$ be a Lipschitz domain in $M$ and consider a function $v \in$ $B_{\lambda}^{p, p}(\Omega)$, for some $\lambda>0$ and $1<p<\infty$. Fix two parameters $\alpha>0, \tau>0$ satisfying

$$
\alpha / d>\max \{0,1 / \tau-1\}
$$

and either

$$
\frac{1}{\tau}>\frac{1}{p}, \quad \alpha-\frac{1}{\tau}<\lambda-\frac{1}{p}
$$




$$
\alpha<\lambda, \quad \frac{1}{\tau}-\frac{\alpha}{d} \geq \frac{1}{p}-\frac{\lambda}{d}
$$

and pick an integer $m$ such that

$$
m>\lambda-\frac{1}{p}+\frac{1}{\tau}
$$

Also, assume $\nabla^{m} v \in L_{\mathrm{loc}}^{1}(\Omega)$ and

$$
\operatorname{dist}(\cdot, \partial \Omega)^{m-\lambda}\left|\nabla^{m} v\right| \in L^{p}(\Omega)
$$

Then it follows that $v \in B_{\alpha}^{\tau, \tau}(\Omega)$.

Note that the assumptions (2.1)-(2.3) made on $\alpha$ and $\tau$ amount precisely to the requirement that the point with coordinates $(\alpha, 1 / \tau)$ belongs to the region $\Theta^{d}(\lambda, 1 / p)$.

Proof. The case when (2.3) is satisfied follows directly from well-known embedding theorems. Thus, henceforth we shall assume that (2.2) holds. In particular, $\tau \in$ $(0, p)$.

Next, since the problem is local in character, there is no loss of generality in assuming that $\Omega$ is a bounded, Euclidean Lipschitz domain. In this context, we shall closely parallel the arguments in the proof of Theorem 3.2 in [2]. In fact, in the interest of brevity, we shall adopt the notation utilized in 2, sketch the main steps and only insist on the modifications which become necessary as a result of dropping the hypothesis $1 / \tau=\alpha / d+1 / p$ which was enforced throughout [2].

Consider a sufficiently smooth wavelet basis, generated via the usual dilationtranslation procedure by a family of functions $\eta \in \Psi$, compactly supported in some large cube $Q$ centered at the origin. Extending $v$ to $B_{\alpha}^{p, p}\left(\mathbb{R}^{d}\right)$ and relying on a characterization of membership to the Besov scale in terms of wavelet coefficients (5], 7]), the goal is to show that

$$
\begin{aligned}
& \sum_{\eta \in \Psi} \sum_{I \in \mathcal{D}^{+}}|I|^{-\alpha \tau / d}\left|\left\langle v, \eta_{I, \tau^{\prime}}\right\rangle\right|^{\tau} \\
& \quad=\sum_{\eta \in \Psi} \sum_{I \in \mathcal{D}^{+}}|I|^{\tau(1 / \tau-1 / p-\alpha / d)}\left|\left\langle v, \eta_{I, p^{\prime}}\right\rangle\right|^{\tau}<\infty .
\end{aligned}
$$

Here $\mathcal{D}^{+}$is the collection of all dyadic cubes $I$ of volume $|I| \leq 1,1 / \tau+1 / \tau^{\prime}=1$ and $\eta_{I, \tau^{\prime}}$ stands for $|I|^{1 / 2-1 / \tau^{\prime}} 2^{j d / 2} \eta\left(2^{j} \cdot-k\right)$ if $I=2^{-j}\left(k+[0,1]^{d}\right), k \in \mathbb{Z}^{d}, j \in \mathbb{Z}$, $\eta \in \Psi$.

Compared to [2], the factor $|I|^{\tau(1 / \tau-1 / p-\alpha / d)}$ in [2.6) is new and our aim is to monitor its effect on subsequent calculations. Let $\Lambda_{j}^{o}$ be the subset of $\mathcal{D}^{+}$consisting of dyadic cubes $I=2^{-j}\left(k+[0,1]^{d}\right) \in \mathcal{D}^{+}$such that $Q(I):=2^{-j}(k+Q)$ is contained inside $\Omega$ and lies at a distance $\geq 2^{-j}$ to the boundary. When the range of summation 
in (2.6) is restricted to $\Lambda_{j}^{o}$, it follows that $|I|=2^{-j d}$ so that

$$
\begin{aligned}
& \sum_{\eta} \sum_{I \in \Lambda_{j}^{o}}|I|^{\tau(1 / \tau-1 / p-\alpha / d)}\left|\left\langle v, \eta_{I, p^{\prime}}\right\rangle\right|^{\tau} \\
& \leq C 2^{[-j d \tau(1 / \tau-1 / p-\alpha / d)-j m \tau]}\left(\sum_{I \in \Lambda_{j}^{o}} \operatorname{dist}(Q(I), \partial \Omega)^{p \tau(\lambda-m) /(p-\tau)}\right)^{(p-\tau) / p} \\
& \quad \times\left(\int_{\Omega}\left|\operatorname{dist}(\cdot, \partial \Omega)^{m-\lambda}\right| \nabla^{m} u||^{p}\right)^{\tau / p} \\
& \leq C 2^{[-j d \tau(1 / \tau-1 / p-\alpha / d)-j m \tau]}\left(\sum_{k=1}^{C 2^{j}} 2^{j(d-1)}\left(k 2^{-j}\right)^{p \tau(\lambda-m) /(p-\tau)}\right)^{(p-\tau) / p} \\
& \leq C 2^{-j \tau(\lambda-\alpha+1 / \tau-1 / p)}\left(\sum_{k=1}^{\infty} k^{p \tau(\lambda-m) /(p-\tau)}\right)^{(p-\tau) / p}
\end{aligned}
$$

This sequence of inequalities can be justified as in [2], based on classical Whitney estimates and Hölder's inequality. The important observation is that, thanks to the second inequality in (2.4), the exponent of $k$ above is $<-1$. Hence, the last series above converges, leading to an overall bound of the order $2^{-j \tau(\lambda-\alpha+1 / \tau-1 / p)}$. Now, since $\sum_{j=0}^{\infty} 2^{-j \tau(\lambda-\alpha+1 / \tau-1 / p)}<+\infty$ (as the first inequality in (2.4) guarantees), the corresponding piece in (2.6) is taken care of.

There remains the part in (2.6) when the sum is performed over the layers $\Lambda_{j, 0}$, $j=0,1, \ldots$, defined analogously to $\Lambda_{j}^{o}$ with the sole exception that, this time, the distance from $Q(I)$ to $\partial \Omega$ is assumed $\leq 2^{-j}$. Once again, this is dealt with much as in 2] but carrying along the factor $2^{-j d \tau(1 / \tau-1 / p-\alpha / d)}$. Ultimately, this yields

$$
\begin{aligned}
& \sum_{j=0}^{\infty} \sum_{\eta \in \Psi} \sum_{I \in \Lambda_{j, 0}}|I|^{\tau(1 / \tau-1 / p-\alpha / d)}\left|\left\langle v, \eta_{I, p^{\prime}}\right\rangle\right|^{\tau} \\
& \leq C\|v\|_{B_{\lambda}^{p, p}(\Omega)}^{\tau}\left(\sum_{j=0}^{\infty} 2^{-j \tau(\lambda-\alpha+1 / \tau-1 / p)}\right)^{(p-\tau) / p} .
\end{aligned}
$$

Note that, due to our assumptions, the last sum above is finite. Thus (2.6) follows and this finishes the proof of the proposition.

Turning attention to layer potentials, we shall need the following.

Proposition 2.2. Let $\Omega$ be a Lipschitz domain in $M$ and consider a positive integer $N$. Suppose that the kernel $k(x, y)$ is defined on $M \times M \backslash$ diagonal and satisfies (1.16). Also, let $\mathcal{K}$ be the integral operator with kernel $k(x, y)$ as in (1.15).

Then, for $1<p<\infty$ and $0<s<1$, this operator satisfies the estimates

$$
\begin{aligned}
\left\|\operatorname{dist}(\cdot, \partial \Omega)^{s+i-1 / p}\left|\nabla^{\theta+i} \mathcal{K} f\right|\right\|_{L^{p}(\Omega)} & \\
& +\sum_{k=0}^{\theta+i}\left\|\nabla^{[\theta+i-k-1]_{+}} \mathcal{K} f\right\|_{L^{p}(\Omega)} \leq C\|f\|_{B_{-s}^{p, p}(\partial \Omega)}
\end{aligned}
$$


for all $i=0,1, \ldots, N-1$, uniformly for $f$ in $B_{-s}^{p, p}(\partial \Omega)$. Here $[a]_{+}:=\max \{a, 0\}$ and $\nabla^{0}$ is the identity. Furthermore, the operator

$$
\mathcal{K}: B_{-s}^{p, p}(\partial \Omega) \longrightarrow B_{\theta-s+1 / p}^{p, p}(\Omega)
$$

is bounded in each of the following situations:

$$
0<s<1 \text { and } \theta \geq 1 \text {, or } \theta=0 \text { and } 0<s<1 / p \text {. }
$$

Proof. The estimate (2.9) has been proved in [11] (which, in turn, builds on the results of [8] and [10]). The claim regarding (2.10) has also been established in [11] for $\theta \in\{0,1\}$ from which the general case needed here also follows.

Proposition 2.3. Let $k(x, y)$ denote the Schwartz kernel of a (classical) pseudodifferential operator $P \in O P S^{-1-\theta}(M)$ satisfying the hypotheses of Theorem 1.4. Then, for each $1<p<\infty$, the operator

$$
\mathcal{K}: L_{-s}^{p}(\partial \Omega) \rightarrow B_{\theta-s+1 / p}^{p, \hat{p}}(\Omega), \quad \hat{p}:=\max \{p, 2\},
$$

is well-defined and bounded provided either $\theta=0$ and $s=0$, or $\theta \geq 1$ and $0 \leq s \leq 1$. Moreover, for $s \in\{0,1\}$,

$$
\operatorname{dist}(\cdot, \partial \Omega)^{1 / 2}\left|\nabla^{1+\theta-s} \mathcal{K} f\right| \in L^{2}(\Omega), \quad \forall f \in L_{-s}^{2}(\partial \Omega) .
$$

Proof. Again, for $\theta \in\{0,1\}$, the claim regarding (2.12) has been proved in [11. Clearly, the case of arbitrary $\theta$ also follows from this. Finally, (2.13) has been obtained in [8] when $s=0$ and the case $s=1$ can be deduced from this (by proceeding as in $\S 3$ of [1]]).

After these preliminaries, we are ready to present the proofs of the main results.

Proof of Theorem 1.1. Under the current assumptions, Lemma 4.5 in 10 gives that

$$
\operatorname{dist}(\cdot, \partial \Omega)^{1-\alpha}|\nabla u| \in L^{p}(\Omega) \text {. }
$$

Interior estimates (cf. Proposition 3.2 in [10] which continues to hold for $s<0$ ) then further yield

$$
\operatorname{dist}(\cdot, \partial \Omega)^{2-\alpha}\left|\nabla^{2} u\right| \in L^{p}(\Omega),
$$

so that (2.5) holds with $m=2$. In particular, granted the current hypotheses, the inequalities (2.4) are satisfied. Then the desired conclusion (in the first part of the statement) follows from Proposition 2.1.

Finally, the second claim in the theorem is proved similarly given that (2.14) holds for any $1<p<\infty$ under the stronger smoothness assumptions (1.7); cf. $\S 3$ in [10].

Proof of Theorem 1.2. For starters, we shall prove that, if $L$ is as in (i), then

$$
\begin{aligned}
& u \in L_{\mu}^{p}(\Omega), 0<\mu<1+1 / p, 1<p<\infty, L u=0 \text { in } \Omega, \\
& \Longrightarrow u \in B_{\mu}^{p, p}(\Omega) \text { and } \operatorname{dist}(\cdot, \partial \Omega)^{1-\mu}|\nabla u| \in L^{p}(\Omega) .
\end{aligned}
$$

Indeed, when $0<\mu<1$ this follows from Lemma 4.5 and Lemma 4.6 in [10]. Thus, it suffices to treat the case when $1 / p<\mu<1+1 / p$. In this situation, the trace operator $\operatorname{Tr}$ is well-defined and $\operatorname{Tr} u \in B_{\mu-1 / p}^{p, p}(\partial \Omega)$. Also, $\partial_{\nu} u \in B_{\mu-1 / p-1}^{p, p}(\partial \Omega)$, where the conormal derivative is defined in the distributional sense by requiring

$$
\left\langle\partial_{\nu} u, \operatorname{Tr} \phi\right\rangle:=\iint_{\Omega}\langle D u, D \phi\rangle, \quad \forall \phi \in\left(L_{\mu-2}^{p}(\Omega)\right)^{*} .
$$


Next, by eventually considering $L-V$ in place of $V$, where $V \in C^{\infty}(M), V \geq 0$, supp $V \subset M \backslash \bar{\Omega}$, there is no loss of generality assuming that $L$ satisfies the following nonsingularity hypothesis

$$
\forall D \subseteq M \text { Lipschitz, } \omega \in H_{0}^{1,2}(D) \text { and } L \omega=0 \Longrightarrow \omega \equiv 0 \text { in } D
$$

and denote by $E(x, y)$ a fundamental solution for the operator $L$, i.e. $E$ is the Schwartz kernel of $L^{-1}$. For more details, as well as an analysis of the nature of the singularity of $E$ along the diagonal in $M \times M$, the reader is referred to 8 . Next, let $\mathcal{S}, \mathcal{D}$ stand, respectively, for the associated single and double layer potentials, i.e.

$$
\mathcal{S} f(x):=\int_{\partial \Omega}\langle E(x, y), f(y)\rangle d \sigma_{y}, \mathcal{D} f(x):=\int_{\partial \Omega}\left\langle\partial_{\nu_{y}} E(x, y), f(y)\right\rangle d \sigma_{y}, \quad x \in \Omega .
$$

Then (2.16) follows from the Green representation formula

$$
u=\mathcal{D}(\operatorname{Tr} u)-\mathcal{S}\left(\partial_{\nu} u\right), \quad \text { in } \Omega,
$$

and the mapping properties of the layer potentials involved. (In the case of $\mathcal{S}$, (1.17) with $\theta=1$ will do; thanks to the work in [10, [9], similar results hold for D.) With (2.16) at hand, the desired conclusion in Theorem 1.2 when $L$ satisfies (i) is proved much the same as in Theorem 1.1, chossing $\mu:=s+1 / p$.

Next, turning attention to the case when $L$ satisfies (ii) in Theorem 1.2 first observe that

$$
u \in B_{s+1 / 2}^{2,2}(\Omega), 0 \leq s \leq 1, L u=0 \text { in } \Omega \Longrightarrow \operatorname{Tr} u \in B_{s}^{2,2}(\partial \Omega) .
$$

Indeed, for $0<s<1$ this is a consequence of the usual trace theorem (cf. [6] for an extension at the level of Lipschitz domains), while the endpoint cases $s \in\{0,1\}$ follow from the work in [8], [10].

Going further, let us assume for a moment that $L$ satisfies the nonsingularity hypothesis (2.18) and recall the single layer potential operator $\mathcal{S}$. It has been proved in [8] that its boundary version, i.e. $S:=\operatorname{Tr} \circ \mathcal{S}$, is invertible from $L_{-\theta}^{2}(\partial \Omega)$ onto $L_{1-\theta}^{2}(\partial \Omega)$ for $\theta \in\{0,1\}$. Thus, by real interpolation,

$$
S: B_{-\theta}^{2, q}(\partial \Omega) \rightarrow B_{1-\theta}^{2, q}(\partial \Omega) \text { is invertible for each } 0<\theta<1,1<q<\infty .
$$

Also, granted the current hypotheses on $u$, the layer potential representation

$$
u=\mathcal{S}\left(S^{-1}(\operatorname{Tr} u)\right)
$$

holds in $\Omega$ (cf. [8]). Now, observe that $S^{-1}(\operatorname{Tr} u) \in B_{s-1}^{2,2}(\partial \Omega)$ and recall from (1.17) with $p=2, \theta=1$, that

$$
\mathcal{S}: B_{s-1}^{2,2}(\partial \Omega) \rightarrow B_{\gamma}^{\tau, \tau}(\Omega) \text { if }(\gamma, 1 / \tau) \in \Theta^{d}(s+1 / 2,1 / 2) \text { and } 0<s<1 .
$$

Hence, granted (2.18), the regularity statement (1.12) follows from (2.23) and (2.24). Dispensing of the extra hypothesis (2.18) can be done by working with $L-t I$ in place of $L$, where $t \in \mathbb{R}$ is a sufficiently large constant, and with $w:=u+t(L-t I)^{-1} u$ in place of $u$. The idea is that $(L-t I) w=0$ and the Newtonian type potential $(L-t I)^{-1}$ is smoothing of order 2 (cf. the discussion in [10]). 
Finally, (1.13), corresponding to the limiting case when $s \in\{0,1\}$, follows along similar lines. This time, however, the version of (2.24) which is needed reads

$$
\mathcal{S}: L_{-\theta}^{q}(\partial \Omega) \rightarrow B_{1-\theta+1 / p}^{q, 2}(\Omega)
$$

for $0 \leq \theta \leq 1,1<q \leq 2$. This, however, is covered by (2.12).

Proof of Theorems 1.3 1.4 These follow more or less directly from Propositions 2.1 2.3. When dealing with Theorem 1.3 one chooses $m:=\theta+N-1$. In the case of Theorem 1.4, one first proves the validity of the cases $s=0$ and $s=1$ in (1.20) $(\theta \geq 1$ is only needed in the former) and then the desired result is obtained by interpolation.

\section{ACKNOWLEDGMENT}

I thank Ron DeVore, who first called this type of phenomenon to my attention, for several stimulating discussions.

\section{REFERENCES}

[1] J. Bergh and J. Löfström, Interpolation spaces. An introduction, Springer-Verlag, 1976. MR 58:2349

[2] S. Dahlke and R. A. DeVore, Besov regularity for elliptic boundary value problems, Comm. PDE, Vol. 22 (1997), 1-16. MR 97k:35047

[3] S. Dahlke, Besov regularity for second order elliptic boundary value problems with variable coefficients, Manuscripta Math., Vol. 95 (1998), 59-77. MR 98m:35038

[4] R. A. DeVore and R.C. Sharpley, Besov spaces on domains in $\mathbb{R}^{d}$, Trans. Amer. Math. Soc., Vol. 335 (1993), 843-864. MR 93d:46051

[5] M. Frazier and B. Jawerth, A discrete transform and decompositions of distribution spaces, J. Funct. Anal., Vol. 93 No. 1 (1990), 34-170. MR 92a:46042

[6] D. Jerison and C. Kenig, The inhomogeneous Dirichlet problem in Lipschitz domains, J. Funct. Anal., Vol. 130 (1995), 161-219. MR 96b:35042

[7] G. Kyriazis, Wavelet coefficients measuring smoothness in $H_{p}\left(\mathbb{R}^{d}\right)$, Appl. Comput. Harmon. Anal., Vol. 3 (1996), 843-864. MR 97h:42016

[8] D. Mitrea, M. Mitrea and M. Taylor, Layer potentials, the Hodge Laplacian and global boundary problems in non-smooth Riemannian manifolds, Memoirs of the Amer. Math. Soc., Vol. 150 No. 713, Providence RI, 2001. CMP 2001:07

[9] M. Mitrea, Generalized Dirac operators on nonsmooth manifolds and Maxwell's equations, The Journal of Fourier Analysis and Applications, Vol. 7 No. 3 (2001), 207-256.

[10] M. Mitrea and M. Taylor, Potential theory on Lipschitz domains in Riemannian manifolds: Sobolev-Besov space results and the Poisson problem, J. Funct. Anal., Vol. 176 No. 1 (2000), $1-79$.

[11] M. Mitrea and M. Taylor, Navier-Stokes equations on Lipschitz domains in Riemannian manifolds, Math. Annalen, Vol. 321 (2001), 955-987.

[12] J. Nečas, Les Méthodes Directes en Théorie des Équations Élliptiques, Academia, Prague, 1967. MR 37:3168

[13] T. Runst and W. Sickel, Sobolev Spaces of Fractional Order, Nemytskij Operators, and Nonlinear Partial Differential Operators, de Gruyter, Berlin, New York, 1996. MR 98a:47071 65211

Department of Mathematics, University of Missouri at Columbia, Columbia, Missouri

E-mail address: marius@math.missouri.edu 